\section{Ensayando recetas para transversalizar la perspectiva de género feminista y los derechos humanos en las prácticas de enseñanza del Derecho Internacional Privado.}

Testing recipes to mainstream the feminist gender perspective and human rights in the teaching practices of Private International Law.

\author{
Erika Silvina Bauger ${ }^{1}$ \\ https://orcid.org/0000-0002-6207-4203 \\ ebauger@jursoc.unlp.edu.ar \\ Facultad de Ciencias Jurídicas y Sociales | \\ UNLP | Argentina
}

\section{RESUMEN}

En estos tiempos de pandemia me propongo abordar la imprescindible incorporación en los procesos de enseñanza y aprendizaje del Derecho Internacional Privado, de la perspectiva de género, los feminismos jurídicos y los derechos humanos como dimensiones transversales a la formación profesional. Estos ejes devienen estratégicos para contribuir a ampliar el campo de los derechos en la sociedad, comprendiendo y explicando cómo operan las desigualdades estructurales imbricadas a tramas de poder. El objetivo del trabajo será reflexionar sobre las prácticas de enseñanza en la Facultad de Ciencias Jurídicas y Sociales de la Universidad Nacional de La Plata y proponer una pedagogía crítica feminista intercultural e interseccional para el diálogo emancipatorio. A los fines didácticos y con el objetivo de pensar en la posibilidad de mejorar los procesos educativos incorporando enfoques críticos a la propia práctica, realizaré un paralelismo entre los elementos de la situación pedagógica de la que habla Freire, con la organización de una cocina profesional de un restaurante.

\footnotetext{
1 Abogada, egresada con Diploma de Honor y Medalla de la UNLP. Premio "Joaquín V. González". Auxiliar Docente con funciones de Adjunta de la Cátedra I de Derecho Internacional Privado de la FCJyS. de la UNLP. Miembro del Instituto de Derecho Internacional Privado del CALP, AADI y ASADIP. Especialización en Derecho Internacional Privado Universidad de Salamanca. Becaria de investigación en Iniciación, Perfeccionamiento y Formación Superior de SECyT de la UNLP. Maestranda en Relaciones Internacionales y en Derechos Humanos de la UNLP. Estudiante de la Especialización en Docencia Universitaria de la UNLP. Docente del Taller de Capacitación Derecho y Lenguaje Claro para el Acceso a la Justicia. Coordinadora y Docente del Seminario "Protección de la Niñez en el Derecho Internacional Privado" y coordinadora y docente del Seminario "Género y Derecho" de la Red de Profesoras de la FCJyS e integrante del Observatorio de Enseñanza del Derecho de la UNLP.
}

\section{PALABRAS CLAVE}

educación superior, prácticas de enseñanza, derecho internacional privado, perspectiva de género, feminismos jurídicos, derechos humanos, interculturalidad. 


\section{KEY WORDS}

higher education, teaching practices, private international law, gender perspective, legal feminisms, human rights, interculturality.

\section{ABSTRACT}

In these times of pandemic, I propose to address the essential incorporation in the teaching and learning processes of Private International Law, the gender perspective, legal feminisms and human rights as transversal dimensions to professional training. These axes become strategic to contribute to broadening the field of rights in society, understanding and explaining how structural inequalities interwoven with power networks operate. The objective of the work will be to reflect on the teaching practices in the Faculty of Legal and Social Sciences of the National University of La Plata and propose an intercultural and intersectional feminist critical pedagogy for emancipatory dialogue. For didactic purposes and with the aim of thinking about the possibility of improving educational processes by incorporating critical approaches to practice itself, I will make a parallel between the elements of the pedagogical situation that Freire speaks of, with the organization of a professional kitchen of a restaurant. 


\section{1) INTRODUCCIÓN}

La Facultad de Ciencias Jurídicas y Sociales de la Universidad Nacional de La Plata, es persistentemente requerida por las demandas sociales y los cambios del contexto, y quienes trabajamos y estudiamos en ella podemos reconocernos o no en ese proceso, habilitando diferentes modos de generar respuestas a cada problema. Siendo esto así, cabe preguntarse: ¿Cuál es la posición que nuestras prácticas universitarias asumen frente a esas realidades? ¿Cómo repensar la docencia, la investigación y la extensión en escenarios intensamente desiguales como el que actualmente se ve profundizado por efecto de la pandemia? ¿Qué papel juegan los derechos humanos y particularmente las dimensiones de género, clase y raza, cuando el objetivo es formar abogados/as críticos/as y comprometidos/as con los problemas de sus pueblos?

Siguiendo a Castro-Gómez (2005), en este contexto, aparece la necesidad urgente de que la universidad empiece a cuestionarse y se piense a sí misma autónoma, crítica y lúcidamente. Esto le permitirá discernir y producir los recursos teóricos del conocimiento a la luz de las necesidades epistemológicas que exige la región.

Como afirma Carosio (2017), detrás de toda cultura está siempre el suelo en el que se habita y este habitar implica el no ser indiferente con lo que allí ocurre. "El arraigo o el "desde dónde" da raíces al nosotrxs y a lo simbólico, el sentido de pertenencia a la tierra y la confianza en la vida son base para el pensar crítico" (p. 18). El pensamiento crítico surge de las experiencias concretas y situadas. Por eso, pensar desde Nuestramérica, como identidad e intención, significa analizar las desigualdades e injusticias que caracterizan a nuestras sociedades. 
Como sostiene Freire (1993), "Toda capacidad científica está al servicio de algo o de alguien, por lo tanto, contra algo o contra alguien" (p. 99). Por ello, un problema siempre supone una opción, no es neutro. Esta opción se encuentra reducida en la universidad: no se discute el para quién ni el para qué del conocimiento, reduciéndose el trabajo docente al cómo operativo e instrumental, que enseña medios para fines que no se debaten.

En este entendimiento, sostenemos con Raffin (2006), que el proyecto civilizatorio occidental originado desde la constitución de los Estados modernos, se ha ido modificando a partir de diversas consideraciones respecto del derecho y en especial, del objeto y fundamentos de los derechos humanos. Los derechos humanos y sus antecesores -los Derechos del Hombre y del Ciudadano (1789) - surgen en la modernidad. Es inútil rastrear genealogías previas, ya que llegan de la mano de la burguesía, del dominio de la naturaleza, de la esperanza en el progreso, del hombre racional y de la dominación y circulación del capital. Es decir, su nacimiento cumplió una función de legitimación de nuevas formas de vida en el Estado moderno y de la configuración de un sujeto de derecho en tanto eje del mundo moderno (Bauger, 2020).

Sin embargo, el estatus históricamente determinado de sujeto de derecho -hombre, mayor, blanco, occidental, cis-heterosexual, sin discapacidades y propietario- escondido dentro del neutro universal de individuo abstracto y autónomo, fue cuestionado y sigue siendo cuestionado, ya que el reconocimiento de derechos a sujetos diversos y, en especial, a las mujeres, sigue siendo dificultoso en la práctica. Como sostiene Guerra Palmero (2010), la igualdad de los "meramente hombres" pasó de ser un argumento de la naciente burguesía ilustrada contra los privilegios estamentales del antiguo régimen, a ser empuñada por todos aquellos y aquellas para los que no se había pensado en su origen: trabajadores, negros, mujeres, homosexuales, pueblos colonizados, etc. La ciudadanía y los derechos, así como la comunidad política, se construyeron a partir de sus "afueras constitutivos".

Es decir, los derechos que nacieron de los privilegios que la burguesía arrancó a los reyes, su lenguaje y sus herramientas, abrieron una doble vía revolucionaria, una segunda brecha de revolución que no había sido prevista por los que construyeron el mundo moderno: la que reclama los sueños no cumplidos de todos, todas y todes de cada uno de sus miembros (Bauger, 2020).

Siguiendo a Salvioli (2009), existen tres niveles de abordaje y análisis posibles en torno a la conceptualización de los derechos humanos y la educación superior: la educación como derecho humano, la educación en derechos humanos, y la práctica de los derechos humanos en el proceso de enseñanza y aprendizaje (entendido éste en sentido amplio, y asumiendo que el mismo comprende todas las situaciones que se gene- 
ran al interior de las instituciones educativas).

Incorporar estos niveles de abordaje posibilita descubrir y fortalecer espacios de acción político-pedagógica indispensables para procesar las demandas de la sociedad y avanzar hacia propuestas educativas que aborden la complejidad de los cambios, alojen lo nuevo y construyan condiciones de igualdad que reconozcan la diversidad y la diferencia de las personas. Visibilizar el papel histórico y político que nuestra Facultad de Ciencias Jurídicas y Sociales tiene en la producción de conocimientos, en los procesos de enseñanza y aprendizaje de las/os futuras/ os abogadas/os y en los vínculos con la sociedad, nos permitirá redefinir los sentidos y la direccionalidad de la educación, su politicidad y los qué, los por qué, los para qué y para quién de la enseñanza.

\section{Visibilizar el papel histórico y político que nuestra Facultad de Ciencias Jurídicas y Sociales tiene en la producción de conoci- mientos, en los procesos de enseñanza y aprendizaje de las/ os futuras/os abogadas/os y en los vínculos con la sociedad, nos permitirá redefinir los sentidos y la direccionalidad de la educación, su politicidad y los qué, los por qué, los para qué y para quién de la enseñanza.}

Dicho esto, cabe interrogarnos por la incidencia, las preguntas, los desafíos que estas construcciones que aquí denominamos transversalización de las perspectivas críticas o feministas del género y los derechos humanos, colocan a las instituciones educativas y los ámbitos de producción de conocimiento científico.

Estos interrogantes también problematizan las prácticas jurídicas de las/os futuras/os profesionales del derecho. Ello por cuanto, el campo jurídico se constituye por actores, actrices e instituciones que pujan por darle sentido al derecho (Bourdieu, 2001). Así, las prácticas reiteradas integran las acciones cotidianas, de tal manera que terminan invisibilizadas en la formación de abogadas y abogados en el ejercicio de la profesión liberal y en la judicatura.

\section{LAS PRÁCTICAS DE ENSEÑANZA DESDE UNA PEDAGOGÍA CRÍTICA FEMINISTA INTERCULTURAL}

Las prácticas de enseñanza reconocen el carácter ético y político. Y en esta visión, aparece la necesidad de "indisciplina" y de liberación de las "jaulas" académicas, en términos de Kaplun (2005), donde el enfoque de derechos humanos y de género, se constituye como una herramienta clave, si queremos posibilitar un encuentro integral, crítico y constructivo entre los diferentes niveles empeñados en la construcción del 
conocimiento, evitando el peligro de utilizar un lugar de enunciación extraño, que reproduzca condiciones de alienación del pensamiento desapegado de la realidad latinoamericana y argentina, y de acuerdo al proyecto civilizatorio occidental que continúa vigente.

Las investigaciones que se viene realizando en nuestra Facultad de Ciencias Jurídicas y Sociales, González y Marano (2014) entre otras, nos permiten situar un ángulo de interrogación y visibilidad respecto de lo que Lagarde y de los Ríos (1996) denomina las cosmovisiones sexo genéricas que integran todas las culturas; y adentrarnos hacia algunos rasgos del ordenamiento patriarcal que aparece naturalizado y en el que se sustentan jerarquías y desigualdades hacia aquellas personas que se alejan del ideal androcéntrico. Orden que instala -al decir de Butler (1999)-, un entramado heteronormativo y una cisnormatividad que penaliza a quienes no se ajustan a dichos mandatos o asumen la disidencia.

Estos desarrollos en constante revisión y confronte, se vinculan estrechamente con movimientos políticos de mujeres y disidencias que desde hace siglos al presente se han organizado en un arco diverso de radicalidad, tramado de experiencias y construyendo formas de interpelación al orden social vigente (examinando y trastocando distintos escenarios e instituciones de la vida social, desnudando los mecanismos y discursos segregativos, las desigualdades y opresiones). En un marco socio histórico abierto y contingente sin garantías, donde encarnan luchas por sociedades más igualitarias y por la ampliación de pisos de derecho.

Las pedagogías críticas no conciben a la enseñanza como un método ni saber separado de las prácticas, por eso para examinar esta dimensión, cabe preguntarnos: ¿Qué hace que una pedagogía sea crítica? Para Giroux (2013) es el reconocimiento de que esta es siempre un intento deliberado por parte de los/as educadores/as de influenciar a través de conocimientos y subjetividades que se producen en los procesos de enseñanza.

Como sostiene Freire (2003), cada persona "es un proceso y un proyecto, y no un destino" (p. 55), y por ello, la complejidad de la práctica educativa es tal, que nos plantea la necesidad de considerar todos los elementos que puedan conducir a un buen proceso educativo, y nos impone la necesidad de inventar situaciones creadoras de saberes, sin las cuales la práctica educativa auténtica no podría darse.

Abordar entonces, la práctica desde una pedagogía crítica feminista supone pensar las prácticas de enseñanza como una actividad intencional (Edelstein, 2000), que genera una situación de asimetría inicial y que imprime explícita o implícitamente algún tipo de racionalidad, expresando un entrecruzamiento de cuestiones de distinto orden. En tal escenario conceptual, se demanda un esfuerzo de indagación acerca de su naturaleza, condicionantes y posibilidades, valorizando la reflexión como construcción crítica de nuestras experiencias, 
lo que implica volver sobre las situaciones, sobre nuestra propia actuación y las racionalidades asumidas acerca de la enseñanza (Bauger y Bidone, 2019).

En esta línea de pensamiento, Ruth Zurbriggen (2013) nos invita a denunciar lo que se levanta como una sólida muralla en las instituciones educativas: la heteronormatividad en los espacios escolares y aquí entendemos que lo dicho alcanza los escenarios universitarios. Propone reconocer que cuerpos, sexualidades, subjetividades, deseos, pasiones, géneros (producidos y reproducidos por una extensa cadena de ficciones) tanto de docentes como de estudiantes, habitan cotidianamente nuestras prácticas en las aulas y se relacionan intrínsecamente con lo que ponemos (¿y exponemos?) a disposición (Zurbriggen (2013, p. 14).

Las prácticas de enseñanza tienen una incidencia inmediata en la generación, reproducción y construcción de sentido de las prácticas de Ixs profesionales de la abogacía. Poseen como notas distintivas ser sociales, contextuadas, históricamente determinadas y situadas dentro de las instituciones (Bauger y Bidone, 2019). En tanto que las prácticas de la enseñanza son sociales, históricas y políticas, se observa que dentro de la propuesta curricular de las instituciones opera un claro recorte de contenidos, seleccionado de un amplio universo de posibilidades. Al respecto, Martínez Bonafé (1998), expresa la posibilidad de cambio sobre el discurso dominante en el currículum por medio del accionar crítico docente, ya que éstos desarrollan su profesión dentro de una sociedad con sus características histórico-políticas.

\section{Las prácticas de enseñanza tienen una incidencia inmediata en la generación, reproducción y construcción de sentido de las prácticas de Ixs profesionales de la abogacía.}

Como exponen Becerra García, Garrido Torres y Romo Beltrán (1989), en el aula hay motivos ocultos, generalmente no presentes en la conciencia de los sujetos de la práctica, que coadyuvan a la permanencia de la relación, la concretizan, la cuestionan y la modifican. En cuanto a la autopercepción del alumno, éste necesita ser reconocido por el docente para constituirse como tal y confirmar su identidad dentro de esa práctica social².

Desde las pedagogías críticas, entendemos que la educación invariablemente responde a vínculos de autoridad que, en relación al saber, no son de dominio o imposición sino de mediación dialéctica entre la explicación, la norma y la utopía (Sacristán, 1978), reconocerlo es central para

2 Al respecto, Perrenoud (1994) habla de "el oficio de alumno", que se desarrolla bajo una serie de normas, reglas, limitaciones y trampas que pueden conducirlo al fracaso. 
configurar aprendizajes relevantes. Ciertamente, los procesos unilaterales de transmisión de información no son procesos de enseñanza ni de aprendizaje. "Saber enseñar no es transferir conocimiento, sino crear condiciones para su propia producción o construcción" (Freire, 1996, p. 32); es ofrecer la posibilidad de pensar más allá de lo establecido y permitir espacios en los cuales docentes y estudiantes pueden comprometerse en el diálogo y la reflexión prospectiva.

Siguiendo a Davini (2008), las teorías constructivistas se alejan de la idea de que quien aprende es un "papel en blanco" (p. 44) y otorgan al alumno el protagonismo del aprendizaje: participa activamente en su construcción, relacionando los nuevos mensajes con las experiencias y conocimientos que tiene almacenados en la memoria. El papel del profesor/a ya no consiste en transmitir información, sino en facilitar y promover el aprendizaje cuyo contenido es construido por el propio estudiante.

Asimismo, el aprendizaje significativo representa para Davini (2008) "un cambio o modificación del comportamiento de carácter duradero y estable" (p. 46). La autora destaca que éste es opuesto al aprendizaje repetitivo, producto de la memorización mecánica. Este aprendizaje es posible cuando quien aprende relaciona las informaciones y el significado del contenido sobre el que trabaja, los vincula con sus conocimientos, significados y experiencias previas, y por ello, los comprende. Ello demanda una intensa actividad participativa de quienes aprenden, reflexionando, debatiendo y descubriendo relaciones.

Ya Freire en el siglo pasado nos aportaba la idea de que el diálogo es la esencia de la tarea de enseñar, idea que busca romper los esquemas verticales característicos de nuestras formas de entender la educación tradicional. La educación, entonces, se hace encuentro, no necesariamente orientado a alcanzar acuerdos. Por el contrario, como confluencia en proceso, que se va concretando a través de la problematización de los contenidos a abordar puede presentarse conflictivo.

En esta línea (Zurbriggen, 2013) explica que no alcanza con ofrecer nuevos contenidos pedagógicamente bien construidos, sino que es fundamental revisar qué enfoques se sostienen consciente o inconscientemente, cómo se enseña, qué modelos pedagógico-didácticos se ponen en acto; $y$ si todo ello cambia o promueve nuevas formas de relacionarse entre las personas, de igual y diferente sexo; más igualitarias, placenteras y satisfactorias.

La apuesta es analizar nuestras propuestas docentes desde una serie de interrogantes, incertidumbres y argumentos que contribuyan a abrir el debate en torno a sentidos instalados y naturalizados sobre la normalidad exorbitante con la que cargamos, para ponerlos en entre-dicho bajo la premisa "no hay normalidad, hay anormalizadores" (Skliar, 2006, p. 196). 
de ser vividas, que todas las vidas tienen estatus de humanidad, que es posible acoger discursos "menos normalizadores acerca de los cuerpos, de los géneros, de las relaciones sociales, de la afectividad y del amor" (Britzman, 2007, p. 18).

Que las y los trabajadores de la educación habitemos una educación más explícita y arriesgada, una comprensión de que la educación es también arriesgar el yo, que nos abramos "a la idea de que algunos riesgos hacen más interesantes a las personas" (Britzman, 2007, p. 18) $y$ a las vidas mismas.

La tarea de enseñar desde una pedagogía crítica tanto en su modalidad presencial como virtual, exige espacios de diálogo, interacción y trabajo en equipo. Y aquí cabe preguntarnos: ¿Qué dinámicas de intercambio ofrecemos? ¿Consideramos los saberes previos de nuestros/as estudiantes? ¿Cómo garantizamos que no sean siempre los/ as mismos/as los que dominan la interacción? ¿Es nuestro modo de ejercer la autoridad posibilitador de diálogos y reconsideraciones? ¿Favorecemos la revisión de nuestras cosmovisiones sexo- genéricas y su reproducción en la explicación, o intervención sobre el estado de cosas? ¿Cuándo interpelamos lo hacemos convocando o forzando a les estudiantes? ¿Cómo evaluamos los procesos de aprendizaje?

\section{¿Favorecemos la revisión de nuestras cosmovisiones sexo- genéricas y su reproducción en la explicación, o intervención sobre el estado de cosas?}

\section{III.- ENSAYANDO CON FREIRE RECETAS PARA TRANSVERSALIZAR EL GÉNERO Y LOS DERECHOS HUMANOS EN LAS PRÁCTICAS DOCENTES.}

Para pensar y repensar nuestras prácticas docentes interpeladas desde una perspectiva de género(s), de derechos humanos y desde los feminismos jurídicos, podemos tomar los elementos de la situación educativa que menciona Freire (2003) en El grito manso. Estos son: a) las/os docentes; b) las/os estudiantes; c) el espacio; d) el tiempo; e) los contenidos curriculares; f) la direccionalidad de la educación.

A los fines didácticos y con el objetivo de pensar en la posibilidad de mejorar los procesos de enseñanza y aprendizaje incorporando enfoques críticos a la propia práctica, realizaré un paralelismo entre los elementos de la situación pedagógica de la que habla Freire, con la organización de una cocina profesional de un restaurante. A los elementos mencionados incluiré uno más, la institución educativa universidad.

\section{a) Las/os cocineras/os: las y los docentes}

Uno de los oficios más exigentes es, sin duda, el de preparar alimen- 
tos, ya que en él confluyen los más dispares requerimientos: que la comida sea rica, nutritiva, sana, en la temperatura correcta, bien presentada y adecuada al establecimiento. Una tarea nada fácil a la que le tendremos que agregar horarios de trabajo, la mayoría de las veces no compatibles con la vida familiar.

En un restaurante, el equipo de cocina está compuesto por: un Chef de cuisine (Jefe de cocina), responsable de toda la gestión de la cocina. Se encarga de supervisar a los empleados, crear menús y con la asistencia del "gerente del restaurante" establece la planificación de la compra de los ingredientes, entrena a los aprendices, supervisa y mantiene la higiene de las áreas de preparación de alimentos. El Sous-chef de cuisine (Subjefe de cocina) que recibe órdenes directamente del chef de cuisine para la gestión de la cocina y a menudo representa al chef de cuisine cuando él/ella no está presente. El Chef de partie (Jefe de partida) que es el responsable de gestionar una estación dada en la cocina cuando se prepara un plato especial. El Commis (Asistente de cocina), que realiza trabajos específicos en una estación, pero reporta directamente al chef de partie y realiza labores de mantenimiento de la estación que está a su cargo. El Apprenti(e) (Aprendiz) que se trata en muchas ocasiones de estudiantes que están aprendiendo habilidades y conocimientos de la cocina y suelen realizar labores de limpieza o de preparación de espacios.

Cuando una cocinera/o se está formando, debe asumir con humildad que le queda un largo camino por recorrer en esa organización jerárquica y que, mientras más conocimientos, técnicas y destrezas logre incorporar, mejor será su desempeño en el campo laboral.

Esta metáfora puede encajar perfectamente con la formación en la carrera docente cuando se intenta integrar una cátedra universitaria.

Por ello, el primer elemento que debemos analizar es quiénes son las y los cocineros que integran la brigada de cocina, cómo se organizan los cargos y las funciones de cada una/o y cómo se decide el menú que van a ofrecer a los comensales.

Respecto de este tópico, podemos preguntarnos desde una perspectiva de género(s): ¿cuál es el perfil sexo genérico hegemónico del plantel docente en nuestra carrera?, ¿cómo se distribuyen los puestos de poder?, ¿cómo se reparten los cargos y dedicaciones docentes? ¿quién decide los contenidos curriculares de la carrera y de cada cátedra? ¿existe precarización laboral docente? ¿las personas que dan clases ejercen la profesión de abogades, detentan cargos en la judicatura o en los otros poderes del Estado?

podemos preguntarnos desde una perspectiva de género(s): ¿cuál es el perfil sexo genérico hegemónico del plantel docente en nuestra carrera?, ¿cómo se distribuyen los puestos de poder?, ¿cómo se reparten los cargos y dedicaciones docentes? 
En cuanto a la distribución sexo genérica de la cátedra de Derecho Internacional Privado en la que me desempeño, cabe señalar que está compuesta por la profesora titular y 3 profesores adjuntos, 1 jefe de trabajos prácticos, 4 auxiliares docentes con funciones de adjunto y 3 adscriptas. La historia de la cátedra muestra un claro predominio masculino en su titularidad. ${ }^{3}$ Asimismo, se observa que no se producen recambios de personas, sino que, por el contrario, se repiten sus gestiones, que se puede afirmar son vitalicias. Estas circunstancias son significativas a la hora de analizar las prácticas educativas que implican una transmisión de subjetividades y la urgencia de evaluar la implementación de un sistema de concursos transparentes, periódicos y con paridad en la composición de los jurados que deben evaluar la clase de oposición. Garantizar estas condiciones es un paso fundamental si queremos tener cátedras democráticas e inclusivas.

Un aspecto que impacta directamente en la distribución de los cargos, se vincula con la ausencia de contemplaciones, adecuaciones y conciliaciones del trabajo en la cátedra con las cargas reproductivas y de cuidado de las integrantes del equipo ${ }^{4}$. Esto se explica, a mi modo ver, en que la distribución de roles en base al sistema sexo/ género tiene un anclaje histórico que se remonta a los inicios de la constitución de los Estados modernos y la imposición del capitalismo como forma de ordenar la sociedad. Esta distribución de roles, se perpetuó a lo largo de la historia impactando en la situación económica de ambos sexos ${ }^{5}$. La distribución desigual se explica en función de este sistema, donde las mujeres o personas feminizadas dedican casi el doble de tiempo que los varones a los trabajos "invisibles" (Bauger, 2020, p. 360) ${ }^{6}$. Esto se profundiza en el contexto actual de pandemia y confinamiento obligatorio?.

3 Se encuentra reseñada por la profesora titular en la página web http://www.catedradip1laplata.com/index.php/la-catedra/resena-historica. En la página se indican quiénes han sido los profesores titulares de la cátedra I de Derecho Internacional Privado (JURSOC - UNLP): Carlos María Vico (1926); Carlos Alberto Lazcano (1943); Huberto María Ennis (1948); Carlos Alberto Lazcano (1953); Juan Carlos Smith (1968); Gualberto Lucas Sosa (1979); Juan Carlos Smith (1984); Gualberto Lucas Sosa (1986); Liliana Etel Rapallini (1996); Gualberto Lucas Sosa (2002); Liliana Etel Rapallini (2005- actualidad).

$4 \quad$ Una forma lograr las conciliaciones es que las facultades tengan guarderías y lugares de lactancia y el otorgamiento de un régimen de licencias amplio a otros miembros de la familia. La Facultad de Ciencias Exactas ha tomado esta iniciativa. Infoplatense (2 de diciembre de 2019). Una facultad de la UNLP tendrá la primer guardería para los hijos de estudiantes, docentes y no docentes. Disponible en https://www.infoplatense.com.ar/nota/2019-12-2-9-10-0-una-facultad-de-la-unlp-tendra-la-primer-guarderia-para-los-hijos-deestudiantes-docentes-y-no-docentes.

$5 \quad$ Históricamente se ligó la vida de la mujer al ámbito privado y al trabajo reproductivo y la del hombre al ámbito público y al trabajo productivo. Con el tiempo, la mujer fue ganando lugar en el mercado laboral, pero sin participar de modo igualitario con los varones. El trabajo "invisible" no remunerado (quehaceres domésticos, apoyo escolar y cuidados), tanto en participación como en intensidad, tienen actualmente un claro predominio femenino. En Bauger, E. S. (2020). El amparo colectivo para garantizar el derecho de igualdad laboral y no discriminación en razón del género. Anales De La Facultad De Ciencias Jurídicas Y Sociales De La Universidad Nacional De La Plata, (50), 049. https://doi.org/10.24215/25916386e049

6 Pueden consultarse las estadísticas en https://www.ilo.org/global/about-theilo/multimedia/ maps-and-charts/enhanced/WCMS_721352/lang--es/index.htm

$7 \quad$ Sobre estas desigualdades de género y COVID se puede consultar la entrevista del 2 de julio de 2020 a la Dra. Diana Maffía: https://ar.radiocut.fm/audiocut/diana-maffia-filosofa-y-especialista-en-temas-genero-en-lopeor-ya-paso/ 


\section{Un aspecto que impacta directamente en la distribución de los cargos, se vincula con la ausencia de contemplaciones, adecua- ciones y conciliaciones del trabajo en la cátedra con las cargas reproductivas y de cuidado de las integrantes del equipo}

Dicho esto, podemos afirmar que las prácticas docentes están sometidas a múltiples tensiones e interferencias que producen desplazamientos de aquello que le es propio. Compartimos con Achilli que las prácticas son:

(...) el trabajo que el maestro desarrolla cotidianamente en determinadas y concretas condiciones sociales, históricas e institucionales, adquiriendo una significación tanto para la sociedad como para el propio maestro, trabajo que si bien está definido en su significación social y particular por la práctica pedagógica, va mucho más allá de ella al involucrar una compleja red de actividades y relaciones que la traspasa, las que no pueden dejar de considerarse si se intenta conocer la realidad objetiva vivida por el docente (...). Estas actividades y relaciones que cotidianamente el maestro despliega fuera del aula, trascienden la práctica pedagógica e inciden sobre ésta y suele perturbarla, alejando al docente de la actividad que le da especificidad a su trabajo: la enseñanza (Achilli, 1986, p. 6).

El análisis entonces, de lo que hacemos las y los docentes en nuestras prácticas de la enseñanza es una tarea problemática no sólo porque constituyen fenómenos sociales complejos en los que se ponen en juego una multiplicidad de variables, sino porque poner la mirada en ellas implica volver a mirarse a una misma, ya que de alguna manera siempre somos actrices protagónicas en ese escenario pedagógico.

\section{b) Las/os comensales: las/os estudiantes}

El menú con las recetas que se ofrezcan tiene el eje en otro/a, que es el/la estudiante que sería -en esta metáfora- el comensal. Nuestros comensales esperan recibir un menú que los entusiasme y los satisfaga. No olvidemos que se come por necesidad fisiológica, pero en cada comida hay una expectativa de placer y evocación que debe ser cumplida porque esa es la esencia del gourmet, la persona para quien trabajamos.

Si el eje entonces es la alteridad y el docente existe para el alumno, tenemos que aceptar que "el otro", el alumno, piensa, siente, toma posiciones y tiene derecho a defenderlas, para crear las condiciones para un aprendizaje emancipador. Poner en juego las diversas inteligencias, valorizar sus trayectorias, sus conocimientos previos, nos lleva a reflexionar sobre la obra de Rancière (1987) en la cual enfatiza la importancia del aprendizaje entre iguales, donde en su relato el texto es un punto de encuentro para la emancipación. Como sostiene Mac- 
kinnon (2005), debemos evitar la motivación por miedo y la infantilización de Ixs estudiantxs, porque si no, estxs aprenden a no respetar sus pensamientos: "se les enseña la jerarquía, la deferencia y el poder, y se los recompensa cuando dominan los códigos necesarios para pertenecer y adaptarse" (p. 173).

Asimismo, debemos tender puentes con la realidad, enriqueciendo al sujeto como ser cultural que identifique y ponga en valor lo que ya sabe. Si no somos capaces de valorizar la alteridad (que incluye tener en cuenta cuestiones sociales, económicas, culturales, de género, etc.), corremos el riesgo de que como dice Rancière (1987) "los excluidos del mundo de la inteligencia suscriben por sí mismos al veredicto de su exclusión" (p.13).

Siguiendo a Freire (2003), cuanto más pensamos en qué es enseñar, qué es aprender, tanto más descubrimos que no hay una cosa sin la otra, que los dos momentos son simultáneos, que se complementan, de tal manera que "quien enseña aprende al enseñar, y quien aprende enseña al aprender" (p. 47). En este marco también es importante buscar dónde está puesto el interés de nuestrxs estudiantes para así crear mejores condiciones para la apropiación del conocimiento: no subordinarlxs ni dominarlxs, sino motivarlxs e interesarlxs.

\section{En este marco también es importante buscar dónde está pues- to el interés de nuestrxs estudiantes para así crear mejores con- diciones para la apropiación del conocimiento: no subordinar- Ixs ni dominarlxs, sino motivarlxs e interesarlxs.}

En cuanto al perfil sexo genérico del estudiantado, las estadísticas oficiales muestran la participación de las mujeres en carreras de pregrado, grado en las distintas regiones en las variables: estudiantes mujeres (58\%), nuevas inscripciones de mujeres $(58,4 \%)$ y egresadas mujeres $(61,1 \%)$ entre los años 2018 y 2019 . En particular, las mujeres estudiantes, inscriptas y egresadas de pregrado y grado se dedican a ramas de estudio vinculadas a las Ciencias Sociales, donde se ubica la carrera de Abogacía, donde hay una mayor presencia de las mujeres que en carreras vinculadas a Ciencias Básicas y Ciencias de la Salud. En carreras de posgrado también se advierte una mayor presencia de mujeres: estudiantes mujeres en un $58,3 \%$, estudiantes inscriptas en un $58,1 \%$ y egresadas en un $57,8 \%$. También se constata que las mujeres estudian carreras vinculadas a las Ciencias Sociales y a las Ciencias Humanas.

En suma, de las estadísticas analizadas y de la propia experiencia docente, el perfil de estudiante que se advierte en mi Facultad de Ciencias Jurídicas y Sociales de la UNLP es predominantemente femenino. A lo que cabe aclarar y denunciar es que nunca tuve alumnxs de la comuni- 
dad LGBTIQ+, lo que muestra la invisibilización, desigualdad y discriminación de géneros.

\section{c) El gerente del restaurante: la institución universitaria}

Hoy en día la universidad convive con otras agencias que trabajan y abordan la educación y las prácticas educativas. Ante las nuevas generaciones y sus formas de contactarse con el conocimiento, el docente debe crear las condiciones de enlace, ya que la institución educativa no es la única que nuclea la práctica educativa, los medios también las movilizan.

En ellas comprende las "políticas administrativas" que abarcan prescripciones y orientaciones en diferentes contextos de regulación. Desde nuestra experiencia personal, dar respuesta a las exigencias de estas políticas burocráticas (SIGEVA, CONICET, CIC, SICYT, etc.), que incluye la categoría "discursos" de Martínez Bonafé (1998), nos sitúa en una posición vulnerable ante estos requerimientos técnico-administrativos, que se encuentra muy lejos de nuestra tarea en el aula. El autor habla de la presión ejercida sobre los docentes desde el discurso profesionalista, que en última instancia refuerza el discurso liberal.

El carácter institucional y público de la práctica de enseñanza le da una dimensión política, ya que la enseñanza se maneja en la esfera de lo público inclusive cuando se desarrolle en una institución privada. Relacionado con lo antes dicho, Achilli (1990) aporta la idea de burocratización en los aprendizajes, debida en parte a las políticas de formación de docentes, donde en ocasiones éstos asisten a cursos "impuestos" con el objetivo de permanecer en el sistema, conformando parte de un proceso de enajenación que deja en "manos del especialista" ciertos temas.

La incidencia de "las agencias" como instancias organizativas de determinación en los diferentes niveles de concreción curricular, abarcando la administración y control, la elaboración y comercialización de material curricular y la producción de conocimiento pedagógico especializado, condicionan también nuestra tarea docente. En nuestro caso un ejemplo es la exigencia de publicar en ciertas revistas indexadas (con referato extranjero), vinculada con nuestra supervivencia en el sistema universitario.

Las estadísticas aportadas en las páginas oficiales ${ }^{8}$ nos ilustran una distribución de cargos en las universidades nacionales en 2019 de docentes varones en un $49,95 \%$ y mujeres en un $50,05 \%$. En cuanto a la distribución por género y categorías (exclusiva, semi-exclusiva y simple), se advierte que los titulares de cátedra con dedicación exclusiva y semi exclusiva son en su mayoría varones: dedicación exclusiva: $55,1 \%$ y semiexclusiva: $55,4 \%$. Se advierte en la estadística y en la práctica docente la 
ausencia en la Facultad de Ciencias Jurídicas y Sociales de la UNLP de la comunidad LGBTIQ+, lo que no se condice con la realidad social.

En cuanto a las autoridades superiores, los cargos se distribuyen $41,42 \%$ masculinos y $58,58 \%$ femeninos. Lo más importante es la asignación de los cargos jerárquicamente superiores que son concedidos preponderantemente a varones: Rector-Presidente en un $87 \%$, Vicerrector $89 \%$, Secretario de la Universidad 65\%, Decanos en un $66 \%$ y Vice Decano 53\%.

Aquí también se advierte, la ausencia en la estadística y en la práctica en la Facultad de Ciencias Jurídicas y Sociales de la UNLP de mujeres y de personas LGBTIQ+ en los puestos jerárquicamente superiores. Es importante resaltar que en nuestra Facultad nunca fue designada una mujer como Decana. Pudiendo concluir que quienes se ubican en los puestos de poder en la política universitaria son los varones cis género.

\section{Aquí también se advierte, la ausencia en la estadística y en la práctica en la Facultad de Ciencias Jurídicas y Sociales de la UNLP de mujeres y de personas LGBTIQ+ en los puestos jerár- quicamente superiores. Es importante resaltar que en nuestra Facultad nunca fue designada una mujer como Decana.}

En suma, quiénes tienen la sartén por el mango y cortan el bacalao son predominantemente varones cis género. La determinación de lo que se hace, quién lo hace y para qué se hacen las cosas en la política universitaria llevan impresas la marca del gerente del restaurante.

\section{d) El espacio y el tiempo}

Respecto de estos elementos, podemos afirmar que la enseñanza es una práctica social en la que participan sujetos sociales con constituciones identitarias $y$, además, porque se dan juegos de representaciones sociales y autorreferenciales que se acontecen en un tiempo y en un espacio. Respecto a lo que sucede en el aula, cabe preguntarnos ¿cuánto conocemos a nuestras/os estudiantes?

En esta línea de análisis, y siguiendo a Edelstein (2019), conviene resaltar la existencia de dos procesos importantes que debiéramos identificar: 1) el metacognitivo, donde el análisis se centra en nuestro proceso de aprendizaje y 2) el metaanalítico, donde el análisis consiste en colocar una transparencia para entender lo que acontece en el proceso en el que estamos participando. Por ejemplo, registrando lo que pasa en la clase, si hay bullicio y se necesitaba un respiro a través de un recreo.

La cuestión central es trabajar la mirada, la escucha y ampliar los registros de lo que pasa en el aula. Hay que desnaturalizar, deconstruir lo que vimos como natural y normal para poder incorporar otra cosa. 
Tenemos que estar alertas a si los estudiantes están cansados, vigilando el clima del aula; la antropología nos propone analizar los gestos, las señales, los indicios que nos permiten construir pistas de lo que pasa en el aula (Achilli, 1990).

En este sentido traemos a colación a Jackson (1992) cuando reconoce la inmediatez de los acontecimientos en el aula, propiciando observar las manifestaciones del alumno que nos den una pista sobre el trabajo que él está desarrollando; asimismo Romo Beltrán, Becerra García y Garrido Flores (1989) dicen que "La observación implica asistir con teoría, y apertura...," debe ejercitarse la habilidad de observación al tiempo que debe existir deseo de interpretar lo que sucede en el aula. Respecto a estos conceptos, nos surge pensar en el tema de la "inclusión" y la necesidad de incorporar la perspectiva de la discapacidad en la enseñanza. En el caso de la cátedra I de Derecho Internacional Privado, del último año de la carrera de Abogacía, cursó un alumno con una discapacidad visual total, que llegó a esta instancia avanzada de la carrera mediante el uso de un grabador en el que registraba las clases; En estos tiempos de pandemia y de uso coactivo de clases virtuales, me pregunto ¿cuántos alumnxs habrán quedado en el camino?

\section{e) Los contenidos curriculares: el menú}

Quien decide el menú curricular y gestiona la cocina es el Chef de cuisine, Se encarga de supervisar a los empleados, crear menús y con la asistencia del "gerente del restaurante" establece la planificación de la compra de los ingredientes, entrena a los aprendices, supervisa y mantiene la higiene de las áreas de preparación de alimentos. El Chef puede elegir obturar la participación en las decisiones sobre las recetas del menú con el resto del equipo, o, por el contrario, puede democratizar las decisiones dando espacio a otras miradas y pareceres más frescos e innovadores.

Siguiendo a Edelstein (2019) podemos afirmar que la práctica de enseñanza tiene una especificidad en torno al conocimiento y se traduce en una propuesta curricular; son saberes y conocimientos que están en el marco de un programa más amplio como lo es el currículum, que comprende enfoques teóricos y metodológicos. En muchas ocasiones se registra un corrimiento de esa especificidad, como producto de las tensiones más o menos objetivables del contexto macrosocial (Edelstein, 2019).

Respecto a los saberes, las perspectivas decoloniales y deconstructivistas se preguntan: “¿quién define el saber erudito o sabio?", poniendo así en tensión el fundamento de la enseñanza. Edelstein (2019) Cuestionar los saberes sabio y erudito es el punto de partida para analizar los debates que se están dando en las ciencias de la educación.

Asimismo, en la actualidad están proliferando perspectivas desde las neurociencias y la inteligencia emocional que desarrollan nuevos 
contenidos sobre las determinaciones en el saber a la hora de ejercer una vigilancia epistemológica. En este sentido Recalcati (2016) en "La hora de clase. Por una erótica de la enseñanza", señala a la hora de la clase como el único lugar en el que se supera la falsa antítesis entre conocimientos y competencias, y en el que puede prender el chispazo de la pasión por el saber.

Vinculado a estos conceptos, se encuentra el de transposición que se usaba mucho para hablar de los recaudos epistemológicos de cómo se va generando el pasaje del saber sabio o erudito al saber de enseñar, para convertirlo en un saber enseñado, y finalmente el saber apropiado o aprendido por los estudiantes. De tal forma, la traducción (para algunos autores sinónimo de transposición) nunca implica una copia fiel sino aproximaciones sucesivas (Berisso, 2015).

Como exponen Becerra García, Garrido Torres y Romo Beltrán (1989), la institución educativa unge con distintas posiciones a los distintos sujetos intervinientes en la práctica educativa, quedando el/la docente en posesión del saber y por lo tanto en un lugar de poder, mientras que el/a alumno/a se sitúa en uno de dependencia y sometimiento, posiciones que se mantienen por la existencia del acto evaluativo.

En esta interacción entre docente y estudiantes, la enseñanza y aprendizaje habitual de las materias del currículo de la carrera de abogacía, se basa en un modelo científico académico fundado en una falsa neutralidad, desapegado de la vida social y de los problemas concretos de las personas. Esa ausencia de valoración y la "neutralidad" del derecho traen como consecuencia discriminaciones directas e indirectas, de jure y de facto, que exigen trabajar en la transversalización de estudios de género, feminismos jurídicos y derechos humanos en clave intercultural e interseccional, en todas y cada una de las materias del plan de estudios de la carrera (Bauger, 2019), como forma de disputar los sentidos en el campo jurídico y "el derecho a decir al derecho" de actores, actrices e instituciones (Bourdieu, 2001). El objetivo final es que las/os egresadas/os se conviertan en factores de cambio en la aplicación de un derecho más justo y en el acceso real a la justicia para todas las personas.

\section{En esta interacción entre docente y estudiantes, la enseñanza y aprendizaje habitual de las materias del currículo de la carrera de abogacía, se basa en un modelo científico académico funda- do en una falsa neutralidad, desapegado de la vida social y de los problemas concretos de las personas.}

Sin embargo, en la actualidad seguimos observando lo que señalan González y Marano (2014): "En la práctica profesional, en muchas ocasiones, las abogados/as nos dejan perplejos/as cuando sus respuestas 
nos remiten más al pasado que al futuro de una sociedad que soñamos sea cada vez más justa y equitativa" (p. 23).

Como estudio previo, analizamos los programas de las materias del plan de estudios de la carrera, que se publican en la página web de la Facultad, que constituyen el "curriculum formal". Estos representan aquello que está prescripto en forma expresa por la institución comprendiéndolo como "una propuesta de los aprendizajes mínimos que se promoverán en un curso, que forma parte de un plan de estudios y orienta las estrategias de trabajo de autoridades, maestros y alumno" (Díaz Barriga, 1997, p. 47). Este examen nos permite constatar la ausencia de contenidos referidos al tema de intervención propuestos, con excepción de la materia "Derechos Humanos" incluida en el nuevo plan de estudios Nro.69.

Aquí y siguiendo a De Alba (1998), es importante destacar que, tanto lo que dice como lo que silencia un programa de estudios, se relaciona directamente con posturas ideológicas de quienes los diseñan y tiene repercusiones directas en las prácticas profesionales de los/as futuros operadores del derecho.

Por lo expuesto, la primera instancia para la propuesta de intervención académica, será instalar la "cuestión de género(s)"y los feminismos jurídicos a través de un seminario cursado de grado, gratuito y abierto a estudiantes y graduados/as de la carrera de abogacía. Es decir, como el artesano, lo primero será instalar una ventana para algo mayor: la incorporación transversal de la perspectiva de género y los feminismos jurídicos en la enseñanza y aprendizaje

Como señala Alicia De Alba, si queremos cambiar el curriculum debemos empezar a considerar su definición:

Por curriculum, se entiende a la síntesis de elementos culturales (conocimientos, valores, costumbres, creencias, hábitos) que conforman una propuesta política- educativa pensada e impulsada por diversos grupos y sectores sociales cuyos intereses son diversos y contradictorios, aunque algunos tiendan a ser dominantes o hegemónicos, y otros tiendan a oponerse y resistirse a tal dominación o hegemonía. Síntesis a la cual se arriba a través de diversos mecanismos de negociación e imposición social. Propuesta conformada por aspectos estructurales-formales y procesales-prácticos, así como por dimensiones generales y particulares que interactúan en el devenir de la currícula en las instituciones sociales educativas. Devenir curricular cuyo carácter es profundamente histórico y no

9 Asimismo, constatamos que sólo en el ámbito del Instituto de Cultura Jurídica se realizan investigaciones y abordajes en la materia, a través de la Especialización para el Abordaje de las Violencias Interpersonales y de Género, para personas con título de Abogacía, Psicología, Trabajador Social, Licenciados en Comunicación, Médico y/o aquellos profesionales que estén en contacto con la problemática sobre el tema de violencia y género de Universidades Nacionales, Privadas o Extranjeras reconocidas. Existen importantes trabajos sobre la temática, compilados en la obra: La formación de abogados y abogadas. Nuevas configuraciones (2014). Compiladores: González, Manuela Graciela y Marano, María Gabriela, que se citan en la bibliografía. 
En la propuesta política-educativa es ineludible entonces, evaluar su viabilidad, los acuerdos con compañeras/os y la posibilidad de influir con la idea. En este sentido y siguiendo a Stella Maris Abate no podemos obviar el análisis de las condiciones materiales y simbólicas requeridas para su despliegue (de allí el criterio de prudencia para configurar y prever su alcance). Una innovación parte del análisis de lo que tenemos, no se da en el vacío. "Las propuestas serán viables en la medida que el qué y el cómo de su implementación se aborden a la vez" (Abate, 2020, p. 7).

En este entendimiento, la propuesta de intervención académica tendrá como aliadas a las docentes de la Red de Profesoras de la Facultad de Ciencias Jurídicas y Sociales y tendrá, en principio, un objetivo modesto y viable, para sentar las bases para la transversalización de una situación de asimetría de poder.

En realidad, se está pensando no en una reforma, sino en otra manera de pensar el quehacer en la Facultad de Ciencias Jurídicas y Sociales de la UNLP, y en particular en la materia Derecho Internacional Privado, que se ubica al final del plan de estudios de la carrera de abogacía y es una de las puertas de salida para el ejercicio de la profesión. De este modo, los procedimientos tendrán que ser coherentes con hacer posible el proyecto de un encuentro auténtico, comprometido, crítico, propositivo y productivo del trabajo académico, capaz de cuestionar y revertir el modelo patriarcal, homocéntrico y colonial que se tiene al frente.

Por ello, las propuestas de una educación jurídica que transversalicen la aplicación concreta de los derechos humanos de las mujeres y otras identidades no hegemónicas en los procesos de enseñanza y aprendizaje, la investigación y la extensión, tienen que buscar un lugar propio. Un pensamiento propio, situado y anclado desde una perspectiva crítica en las particulares y diversas condiciones y situaciones de las personas. $Y$ para este objetivo, es necesario revisar no sólo las premisas contrarias a su posicionamiento, sino, también aquellas con las que concuerde para detectar posibles prejuicios y espacios de vacancia.

\section{Por ello, las propuestas de una educación jurídica que transver- salicen la aplicación concreta de los derechos humanos de las mujeres y otras identidades no hegemónicas en los procesos de enseñanza y aprendizaje, la investigación y la extensión, tie- nen que buscar un lugar propio.}

\section{f) La direccionalidad de la educación}

Un último elemento para reflexionar sobre la situación pedagógica 
es la direccionalidad del acto educativo. Ya que siguiendo a Freire (2003), no hay situación educativa que no apunte a objetivos que están más allá del aula, que no tenga que ver con concepciones, maneras de ver el mundo, anhelos, utopías. Aquí nos interesa discutir por qué y para qué enseñamos lo que enseñamos, y qué tipo de profesional estamos buscando formar, no sólo en su calidad disciplinar, sino en su calidad humana.

Sobre el punto Berisso (2015) desarrolla la perspectiva de la donación. Da cuenta de la pregunta ¿Qué clase de dar es dar clase? partiendo de la conversación con la ética de Levinas (1977) y de la articulación entre filosofía y educación. En el mismo sentido, dar clase además de ser un acto de generosidad es un acto de carácter intencional (Edelstein 2000).

Desde esta perspectiva nos alejamos de la idea del "dictado de clases"; y en estos términos, si la intención es liberar al otro, aparecen inexorablemente las ideas de Freire (1970) y su crítica a la educación bancaria: "La narración, cuyo sujeto es el educador, conduce a los educandos a la memorización mecánica del contenido narrado. Más aún, la narración los transforma en "vasijas", en recipientes que deben ser "llenados" por el educador." El autor enfatiza: "Saber que enseñar no es transferir conocimiento, sino crear las posibilidades para su propia producción o construcción", esta es la idea esencial que nos propone, en oposición a la memorización mecánica y a un papel distante entre el profesor y sus alumnos. En el esquema indeseado del alumno vasija, Becerra García, Garrido Flores y Romo Beltrán (1989) afirman que "El alumno querrá apropiar la lógica y el lenguaje del docente para complacerlo".

\section{la propuesta que aquí se presenta es la transversalización en la situación pedagógica del enfoque de género, de derechos hu- manos y de discapacidad, que pongan en tensión un derecho falsamente presentado como neutro y carente de politicidad .}

Entonces, si el "dar clases" no es una práctica azarosa, sino que está planeada ya que es una propuesta de intervención, en el sentido inter- venir, ya que las propuestas de enseñanza son para mediar en el alumno y el conocimiento, para como expresa Freire (1968) "crear las posibilidades para su propia producción o construcción", la propuesta que aquí se presenta es la transversalización en la situación pedagógica del enfoque de género, de derechos humanos y de discapacidad, que pongan en tensión un derecho falsamente presentado como neutro y carente de politicidad.

La politicidad de la educación demanda vehementemente de los profesores y de las profesoras que se asuman como un ser político, que se 
descubran en el mundo como un ser político y no como un mero técnico o un sabio, porque también el técnico y el sabio son sustancialmente políticos. La politicidad de la educación exige que el profesor se reconozca, en términos o en nivel objetivo, en el nivel de su práctica, a favor de alguien o en contra de alguien, al favor de algún sueño y, por lo tanto, contra cierto esquema de sociedad, cierto proyecto de sociedad. Por eso entonces la naturaleza política de la educación exige del educador que se perciba en la práctica objetiva como participante a favor o contra alguien o alguna cosa. La politicidad exige del educador que sea coherente en esta opción (Freire, 2016, p. 39).

La politicidad es entonces inherente a la práctica educativa. Esto significa que como profesora debo tener claras mis opciones políticas, mis sueños. Porque, al final, ¿qué es lo que me mueve y me alienta como profesora, si gano tan poco, si estoy tan desprestigiada en esta sociedad de mercado? ¿Qué sueño tengo para soñar, para discutir con mis alumnos? 


\section{BIBLIOGRAFÍA}

Abate, Stella Maris (2020). Apunte: Consignas para pensar y escribir propuestas de intervención académica. Universidad Nacional de La Plata. Especialización en Docencia Universitaria. Taller de Práctica de Intervención Académica.

Achilli, Lidia Elena (2000). Investigación y formación docente. Rosario, Argentina: Laborde Editores. Facultad de Humanidades y Artes de la Universidad Nacional de Rosario.

Bauger, E. S. y Bidone, A. R. (2019). Las prácticas de enseñanza. Especialización en Docencia Universitaria de la Universidad Nacional de La Plata. Disponible en http://sedici.unlp.edu.ar/handle/10915/77410

Bauger, E. S. (2019). Perspectiva de géneros y feminismos jurídicos en la enseñanza del derecho. Derechos En Acción, 11(11), 277.

Bauger, E. S. (2019). Plan de actividades docentes, de investigación y extensión universitarias en Derecho Internacional Privado. http://sedici.unlp.edu.ar/ handle/10915/87876

Bauger, E. S. (2020). El amparo colectivo para garantizar el derecho de igualdad laboral y no discriminación en razón del género. Anales De La Facultad De Ciencias Jurídicas Y Sociales De La Universidad Nacional De La Plata, (50), 049. https://doi.org/10.24215/25916386e049

Bauger, E. S. (2020). Feminismos jurídicos y pluriculturalidad: ensayando una visión en tiempo de pandemia. Derechos En Acción, 16(16), 437. https://doi.org/10.24215/25251678e437

Bauger, E. S. (2020). Propuesta de intervención: Talleres con perspectiva literaria de formación en género y derechos humanos en clave intercultural. Trayectorias Universitarias, 6(11), 043. https://doi.org/10.24215/24690090e043

Becerra García, M. G. y Garrido Flores, M. del R., Romo Beltrán, R. (1989). “De la ilusión al desencanto en el aula Universitaria". En Furlán Alfredo y Pasillas Miguel A. (Comps.) Desarrollo de la Investigación en el campo del currículo. México: U.N.A.M.

Bourdieu, P. (2001). Poder, derecho y clase social. Bilbao: Desclé.

Butler, J. (1999). "Sujetos de sexo/género/deseo". En Gender trouble. Feminism and the Subversion of Identity. Traducción de María Antonia Muñoz: El género en disputa. El feminismo y la subversión de la identidad (pp.45- 85). Barcelona: Paidós. 
Castro Gómez, Santiago (2005). Decolonizar la universidad. La hybris del punto cero y la colonialidad de saberes. En Castro-Gómez S. y Grosfoguel, R. (Comps.), El giro decolonial: reflexiones para una diversidad epistémica más allá del colonialismo global (pp. 79-91). Bogotá: Siglo del Hombre Ed., Universidad Central y Pontificia Universidad Javeriana.

Davini, M. C. (2008). Métodos de enseñanza. Santillana, Buenos Aires.

De Alba, A. (1998). Curriculum: crisis, mito y perspectivas. Argentina: Miño y Dávila editores S.R.L.

Díaz Barriga, Ángel. (1997). Didáctica y Curriculum. Buenos Aires: Paidos.

Edelstein, Gloria (2000). "El análisis didáctico de las prácticas de la enseñanza. Una referencia disciplinar". En Revista IICE. Año IX, № 17. Buenos Aires: Miño y Dávila y Facultad de Filosofía y Letras-UBA.

Freire, Paulo (1970). Pedagogía del oprimido. Montevideo: Tierra Nueva.

Freire, P. (2004). Pedagogía de la autonomía. Saberes necesarios para la práctica educativa. Sao Paulo: Paz y Tierra.

Guerra Palmero, María (2011). "La mujer-filósofo o la más "antinatural" de las criaturas. En torno a Simone de Beauvoir y a su obra El segundo sexo". Revista Valenciana. Estudios de Filosofía y Letras. Núm. 7: enero-junio 2011, pp. 131-146. Recuperado de http://www.revistavalenciana.ugto.mx/index.php/valenciana/ article/view/229

González, M. G. y Marano, M. G. (2014). La formación de abogadas y abogados. Nuevas configuraciones. La Plata: Imás.

Giroux, H. (2013). "La Pedagogía crítica en tiempos oscuros". Praxis Educativa, vol. XVII, núm. 1 y 2, enero-diciembre, 2013, pp. 13-26. UNLPAM. La Pampa, Argentina.

JACKSON, Phillip (1992). La vida en las aulas. Madrid, España: Morata.

Lagarde, M. (1996). "La Perspectiva de género". En Género y feminismo. Desarrollo humano y democracia (pp. 13-38). España: Ed. Horas y HORAS.

Levinas, Emmanuel (1977). Totalidad e infinito. Ensayo sobre la exterioridad. Salamanca, España: Sígueme.

MacKinnon, C. (2005). "Integrando el feminismo en la educación jurídica". Academia. Revista sobre enseñanza del Derecho de Buenos Aires. Año 3, №. 6. 
Martínez Bonafé, J. (1998). Trabajar en la escuela. Profesorado y reformas en el umbral del siglo XX. Madrid, España: Miño y Dávila.

Perrenoud, Philippe (1994). El oficio de alumno y el sentido del trabajo escolar. Madrid, España: Editorial Popular.

Raffin, Marcelo (2006). La experiencia del horror: subjetividad y derechos humanos en las dictaduras y posdictaduras del cono sur. Buenos Aires. Argentina: Editores del Puerto.

Rancière, Jacques (1987). El maestro ignorante. Cinco lecciones sobre la emancipación intelectual. Francia: Laertes.

Recalcati, Massimo (2016). La hora de clase: Por una erótica de la enseñanza. Barcelona, España: Anagrama.

Sacristán, G. J. (1978). "Explicación, norma y utopía en las ciencias de la educación". En Escolano, A. Epistemología y educación (pp.158-167). Salamanca: Sígueme.

Skliar, Carlos (2006). Fragmentos de amorosidad y de alteridad en educación. Revista Colombiana de Educación, núm. 50, enero-junio, 2006, pp. 253266. Universidad Pedagógica Nacional. Bogotá, Colombia.

Zurbriggen, R. (2013) “Cartografiando prácticas y pedagogías feministas en la formación docente (Afectaciones situadas con y sobre cuerpos, géneros, subjetividades y sexualidades)" pp. 12-26 en Siderac, S. (comp.) Educación y género en Latinoamérica. Desafío político ineludible, UNLPAM. 